\title{
Insuficiencia respiratoria y choque séptico por bronquiectasias infectadas. Reporte de un caso
}

\author{
Oscar Antonio Cervantes-González, ** David Alejandro Vargas-Gutiérrez, ** \\ Carmen Margarita Hernández-Cárdenas, * Gustavo Lugo-Goytia*凶 \\ *Unidad de Cuidados Intensivos Respiratorios, Instituto Nacional de Enfermedades Respiratorias Ismael Cosío Villegas,

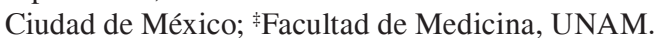 \\ Trabajo recibido: 03-II-2015; aceptado: 17-II-2015
}

\begin{abstract}
RESUMEN. Las bronquiectasias como causa de insuficiencia respiratoria y choque séptico se asocian con una alta mortalidad. Nosotros reportamos el caso de una paciente con una exacerbación aguda por $P$. aeruginosa que le produjo insuficiencia respiratoria severa y choque séptico que requirió manejo en la Unidad de Cuidados Intensivos Respiratorios (UCIR). Cursó con falla multiorgánica y síndrome de debilidad muscular del paciente crítico, requiriendo ventilación prolongada y rehabilitación intensiva. Después de un mes en la UCIR se dio de alta, sin embargo, reingreso una semana después por insuficiencia respiratoria y choque séptico, falleciendo 48 horas después de choque irreversible. Este caso ilustra las alteraciones fisiopatológicas, así como la alta mortalidad a corto plazo en pacientes con bronquiectasias que requieren cuidados intensivos.
\end{abstract}

Palabras clave: Bronquiectasias infectadas, choque séptico.

ABSTRACT. Bronchiectasis as a cause of respiratory failure and septic shock are associated with high mortality. We report the case of a patient with an acute exacerbation due to Pseudomonas aeruginosa that produced severe respiratory failure and septic shock requiring management in the respiratory intensive care unit (RICU). The patient presented multiorgan failure and muscle weakness syndrome requiring prolonged ventilation and intensive rehabilitation. After a month in the RICU the patient was discharged, however she had to be admitted to the unit a week later by septic shock and respiratory failure. The patient died 48 hours after admission by irreversible septic shock. This case illustrates the pathophysiological changes and high short-term mortality in patients with bronchiectasis who require intensive care.

Key words: Infected bronchiectasis, septic shock.

\section{INTRODUCCIÓN}

Las bronquiectasias, definidas como dilataciones permanentes de la pared de los bronquios y bronquiolos, producidas por la infección e inflamación crónica de estas estructuras se caracterizan por un curso crónico, exacerbaciones agudas, deterioro progresivo de la función pulmonar, hipertensión arterial pulmonar y disfunción ventricular., ${ }^{1,2}$ Las principales causas de la formación de bronquiectasias son las infecciones repetitivas del tracto respiratorio, tanto virales como bacterianas que asociadas o no a alteraciones de la movilidad ciliar, inmunodeficiencias, enfermedades reumatológicas o del tejido conjuntivo pueden predisponer a la alteración de la estructura bronquial. Diferentes bacterias se aíslan persistentemente del esputo de estos pacientes, siendo Pseudomona aeruginosa una de las más frecuentes, la cual por medio de la liberación de citocinas proinflamatorias se ha asociado con la más alta morbilidad y mor- talidad. ${ }^{3}$ Nosotros reportamos el caso de una paciente con bronquiectasias que presentó una exacerbación aguda secundaria a $P$. aeruginosa que se complicó con insuficiencia respiratoria severa y choque séptico.

\section{REPORTE DE CASO}

Paciente femenino de 40 años de edad que acudió al Servicio de Urgencias por padecimiento de siete días de evolución caracterizado por fiebre no cuantificada, malestar generalizado, tos productiva con expectoración purulenta y disnea progresiva.

Cinco años antes de su ingreso se le diagnosticó bronquiectasias e hipertensión arterial pulmonar. A partir de entonces cursó con exacerbaciones por infecciones repetidas, sin requerir internamiento en la Unidad de Cuidados Intensivos (UCI).

A su ingreso al Servicio de Urgencias se documentó temperatura de $37.6^{\circ} \mathrm{C}$, frecuencia cardíaca (FC) de 80 
latidos por minuto (Ipm), frecuencia respiratoria (FR) de 45 por minuto, tensión arterial (TA) de 130/70 mmHg. A la exploración física destacó la presencia de cianosis central y periférica, utilización de músculos accesorios de la respiración, estertores basales bilaterales y sibilancias, hepatomegalia, ascitis y edema de miembros inferiores. La radiografía de tórax mostró bronquiectasias basales bilaterales (figura 1). Se inició apoyo respiratorio con ventilación no invasiva, sin embargo, permaneció taquipneica y con dificultad para manejar secreciones, decidiéndose cambiar a ventilación mecánica invasiva (VMI). Posterior a la intubación e inicio de la VMI, después de su tercer día de estancia en el Servicio de Urgencias, se asoció inestabilidad hemodinámica requiriendo apoyo con norepinefrina integrándose un cuadro de choque séptico por lo que se decidió su traslado a la Unidad de Cuidados Intensivos Respiratorios (UCIR).

A su ingreso a la UCIR se realizó broncoscopia con obtención de muestras para cultivos y se ajustó el régimen antimicrobiano de acuerdo con los resultados de éstos. La tomografía computada y la radiografía de tórax se muestran en las figuras 2 y 3 , respectivamente. La evolución respiratoria, hemodinámica y los biomarcadores de función renal y sépsis, así como los resultados de microbiología del exudado bronquial se muestran en la

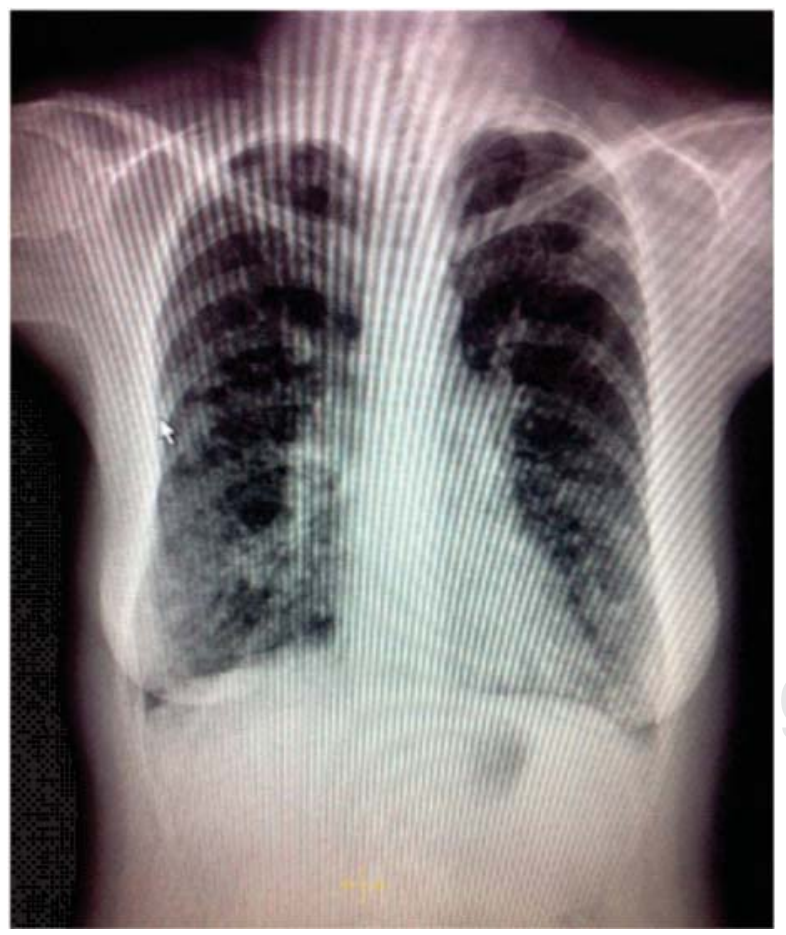

Figura 1. Radiografía de tórax obtenida a su ingreso a urgencias mostrando bronquiectasias basales bilaterales. tabla 1. Durante su evolución la paciente continuó con choque séptico requiriendo dosis altas de vasopresores (norepinefrina y vasopresina) y lesión renal aguda que requirió apoyo con hemodiálisis. Para el día 14 de

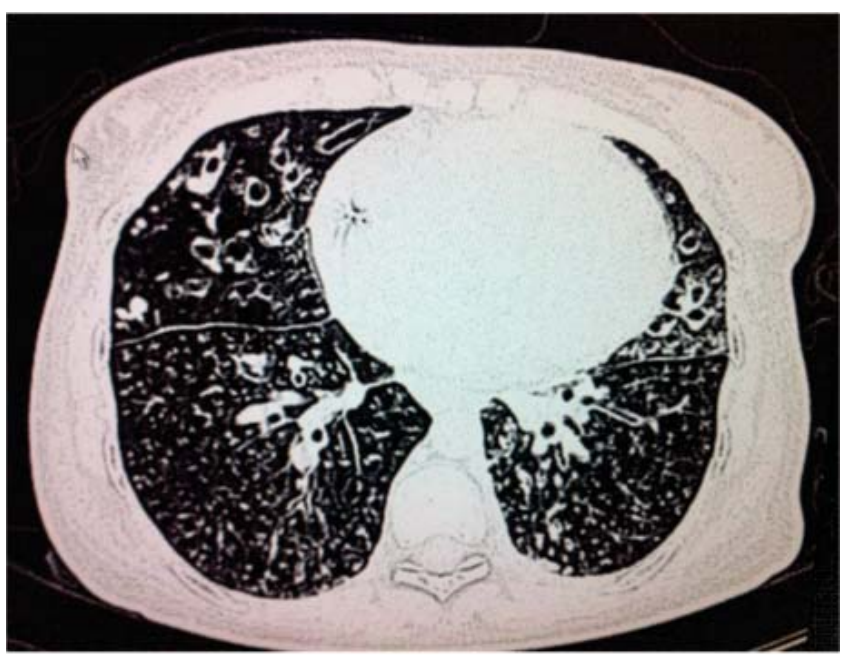

Figura 2. Imagen de tomografía computada de la paciente a su ingreso, la cual muestra múltiples bronquiectasias saculares, engrosamiento de la pared bronquial y secreciones retenidas.

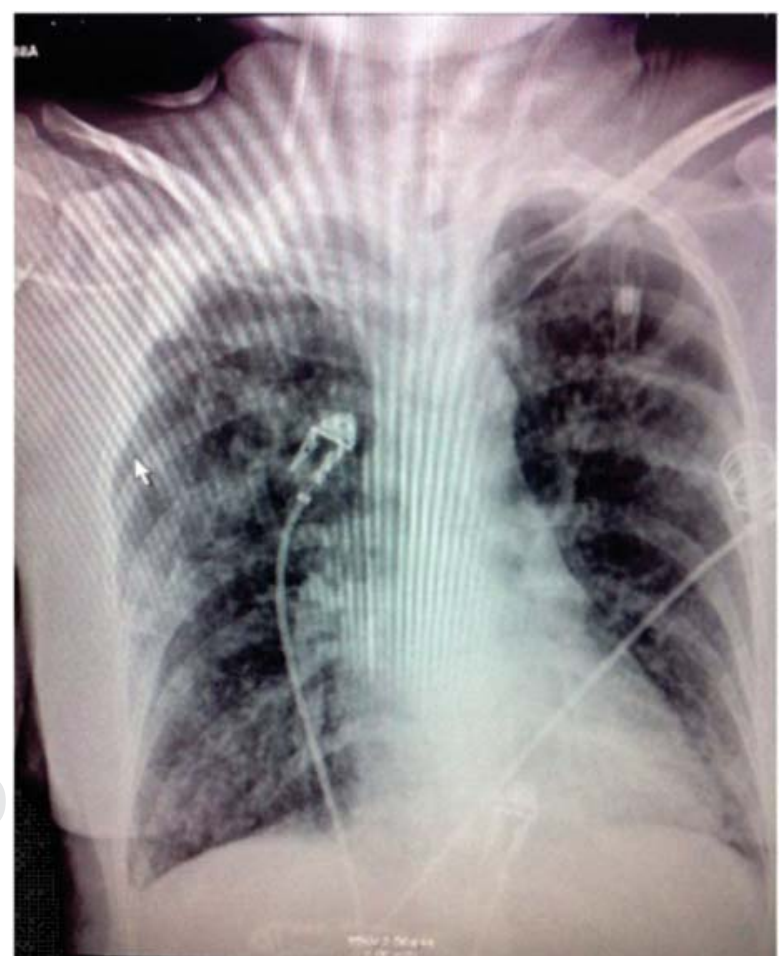

Figura 3. Radiografía de tórax a su ingreso a la Unidad de Cuidados Intensivos Respiratorios la cual muestra las bronquiectasias y la aparición de nuevos infiltrados de predominio derecho. 
Tabla 1. Evolución durante la estancia en la Unidad de Cuidados Intensivos.

\begin{tabular}{|c|c|c|c|c|c|c|}
\hline Día & $\begin{array}{c}\mathrm{PaO}_{2} / \mathrm{FiO}_{2} \\
\mathrm{mmHg}\end{array}$ & $\begin{array}{l}\text { PEEP } \\
\mathrm{cmH}_{2} \mathrm{O}\end{array}$ & $\begin{array}{l}\mathrm{IC} \\
\mathrm{L} / \mathrm{m}^{2}\end{array}$ & $\begin{array}{c}\text { IRVS } \\
\text { CGS }^{*} m^{2}\end{array}$ & $\begin{array}{l}\text { Creatinina } \\
\text { mg }\end{array}$ & $\begin{array}{l}\text { Leucocitos/ } \\
\text { cultivos }\end{array}$ \\
\hline Ingreso & 91 & 8 & 5.06 & 1,440 & 0.9 & $\begin{array}{c}37.6 \text { / P. aeruginosa / panel } \\
\text { viral negativo }\end{array}$ \\
\hline 3 & 204 & 6 & 2.6 & 3,073 & 3.9 & 13.5 \\
\hline 7 & 155 & 6 & 3.46 & 2,596 & 2.61 & 6.8 \\
\hline 14 & 228 & 5 & 4.32 & 2,119 & 1.76 & 7.7 / P. aeruginosa \\
\hline 21 & 327 & 5 & 3.6 & 1,956 & 0.50 & 6 \\
\hline Egreso & 447 & ------ & 4.2 & 1,679 & 0.75 & 6.9 \\
\hline
\end{tabular}

$\mathrm{PaO}_{2}$ : presión arterial de oxígeno; $\mathrm{FiO}_{2}$ : fracción inspirada de oxígeno; PEEP: presión positiva al final de la expiración; IC: índice cardíaco; IRVS: índice de resistencias vasculares sistémicas.

estancia en la unidad, se había controlado el proceso infeccioso y su condición respiratoria y hemodinámica habían mejorado permitiendo su destete de la VMI y extubación. Sin embargo, 24 horas después presentó disnea, aumento del trabajo respiratorio, deterioro de la función renal y congestión pulmonar, motivo por el cual requirió intubación y VMI. Seis días después (día 21) se volvió a estabilizar su condición y se retiró de la VMI exitosamente. Siete días después de su egreso de la UCIR la paciente reingresó debido a insuficiencia respiratoria que requirió nuevamente intubación y VMI debido a un proceso neumónico basal bilateral, evolucionando a choque séptico falleciendo a los dos días de su segundo ingreso a la UCIR.

\section{DISCUSIÓN}

La prevalencia e incidencia de bronquiectasias en nuestro país es desconocida, aunque se sabe que tiende a disminuir al mejorar el acceso a los servicios de salud y de vacunación contra microorganismos como Mycobacterium tuberculosis, Bordetella pertussis, Haemophilus influenzae y sarampión. Seitz et al. ${ }^{4}$ en su artículo sobre la tendencia de bronquiectasias en la población norteamericana durante el período 20002008 reportaron un incremento en la prevalencia de bronquiectasias en la población mayor de 65 años, con predominio en mujeres y en la población asiática. Asimismo, el aumento en el uso de la tomografía de tórax incrementó la prevalencia, por tanto, no concluye si hay un verdadero aumento en la incidencia o se trata de una enfermedad que permanece subdiagnosticada.

Las bronquiectasias representan un tipo específico de lesión bronquial que puede resultar debido a diferentes enfermedades subyacentes. Entre éstas destacan las causas infecciosas como tuberculosis y neumonías recurrentes. Estados de inmunodeficiencia como la hipogamaglobulinemia primaria o secundaria (ej. linfoma o mieloma) o la infección por el virus de la inmunodeficien- cia humana. La aspergilosis broncopulmonar alérgica se presenta en algunos pacientes con asma favoreciendo el desarrollo de bronquiectasias y se asocia con eosinofilia y elevación de lgE. La discinesia ciliar secundaria a infecciones virales, bacterianas y humo de tabaco o primaria como una condición autosómica reciba es causa infrecuente de bronquiectasias. La fibrosis quística es una causa importante de bronquiectasias, esta condición es causada por la mutación del gen regulador de la conductancia a través de la membrana de la fibrosis quística (CFTR), del cual hasta la fecha se han descrito más de 1,800 mutaciones. Su diagnóstico se basa en la demostración de concentraciones elevadas de cloro en el sudor inducido por la iontoforesis de pilocarpina y los hallazgos clínicos característicos como: infecciones respiratorias recurrentes y/o evidencia de insuficiencia pancreática. La etiología de las bronquiectasias en nuestra paciente no se determinó debido a su condición de gravedad. Sin embargo, su condición podría ser consistente con una fibrosis quística no clásica o atípica. En su forma atípica, que se presenta hasta en un $10 \%$, los pacientes tienen un inicio tardío en la vida adulta, habiendo sido diagnosticados previamente como asma, bronquitis crónica o bronquiectasias y por lo regular no manifiestan insuficiencia pancreática. Sin embargo, se sabe que hasta en un $40 \%$ de los pacientes con bronquiectasias no se puede determinar la causa y son clasificadas como idiopáticas.

Las bronquiectasias en nuestra paciente mostraron una morfología quística con una distribución difusa y bilateral de predominio en las bases pulmonares, lo que la hubiese hecho candidata para una evaluación intencionada en búsqueda de enfermedades sistémicas que pudieran condicionar su desarrollo ya que no se conocía con diagnósticos previos asociados a su patología, además, entra dentro del grupo de bronquiectasias con hipertensión arterial pulmonar ya diagnosticada previamente y corroborada en el ecocardiograma de ingreso a la UCIR que mostró cavidades cardíacas derechas 
dilatadas con hipertrofia, así como una presión sistólica pulmonar estimada por arriba de $60 \mathrm{mmHg}$. Alzeer et al., en su artículo sobre la función ventricular y la presión arterial pulmonar en pacientes con bronquiectasias demuestran la existencia de un deterioro sistólico del ventrículo derecho y la presencia de hipertensión arterial pulmonar en los pacientes con bronquiectasias quísticas, además de referir la presencia de disfunción diastólica del ventrículo izquierdo y alteraciones en el intercambio gaseoso en los casos de enfermedad severa. ${ }^{2}$

Por otro lado, el reingreso de nuestra paciente a la $\mathrm{UCl}$ fue posterior a las 48 horas de su traslado al área hospitalización (siete días posteriores) por lo que no se puede considerar como falla al egreso de la $\mathrm{UCI}$ ya que aunque pudo estar condicionado nuevamente por un cuadro infeccioso, en este grupo de pacientes las colonizaciones crónicas de las secreciones bronquiales por bacterias multirresistentes los hacen propensos a constantes recaídas.

El pronóstico de estos pacientes después de su estancia en la $\mathrm{UCl}$ es pobre ya que casi la mitad llegan a fallecer al año de egreso. Dupont et al., ${ }^{5}$ en su artículo de la sobrevida de los pacientes con bronquiectasias infectadas después de su primera hospitalización en la $\mathrm{UCl}$ refiere una mortalidad de $19 \%$ durante la estancia en la $\mathrm{UCl}$ y una mortalidad al año que alcanza hasta el $40 \%$ de los casos, determinando como factores más influyentes una edad mayor a 65 años, el requerimiento de oxígeno suplementario por lapsos prolongados y una puntuación mayor a 32 de la escala SAPS II.

En el manejo del paciente con bronquiectasias con descompensación aguda severa que requiere ingresar a la UCIR son importantes las siguientes medidas terapéuticas: 1. Control del foco séptico pulmonar, inicialmente con antibióticos de amplio espectro que cubran los microorganismos más frecuentes, escalando o desescalando posteriormente de acuerdo con los resultados de los cultivos; 2 . Cotratamiento con antibióticos inhalados (ej., tobramicina, amikacina, colistina); 3. Apoyo respiratorio, inicialmente con ventilación no invasiva y en caso de deterioro ventilación invasiva con ventilación de protección pulmonar, frecuencia respiratoria para evitar auto PEEP; 4. Apoyo cardiovascular con vasopresores y/o inotrópicos para mantener una adecuada perfusión orgánica; 5 . Inicio temprano de soporte nutricional por vía enteral alcanzando requerimientos totales en las primeras 48 a 72 horas y con vigilancia estrecha de niveles de fosforo; 6 . Apoyo de la función renal con hemodiálisis en caso de lesión renal aguda; y 7. Rehabilitación física temprana para prevenir el desarrollo de síndrome de debilidad muscular y permitir un destete temprano de la VMI.

\section{CONCLUSIONES}

El ingreso a la $\mathrm{UCl}$ por choque séptico secundario a bronquiectasias infectadas es una causa poco frecuente pero con alta mortalidad, siendo reportada hasta el $60 \%$ durante el primer año posterior a su estancia. La decisión para admitir a un paciente con bronquiectasias complicadas a la $\mathrm{UCl}$ deberá de basarse en la condición de cada paciente en forma individualizada considerando: su reserva cardiopulmonar, hospitalizaciones previas y expectativa de sobrevida y calidad de vida a corto plazo.

\section{REFERENCIAS}

1. Evans SA, Turner SM, Bosch BJ, Hardy CC, Woodhead MA. Lung function in bronchiectasis: the influence of Pseudomonas aeruginosa. Eur Respir J 1996;9(8):16011604.

2. Alzeer $\mathrm{AH}, \mathrm{Al}-$ Mobeirek AF, Al-Otair HA, Elzamzamy UA, Joherjy IA, Shaffi AS. Right and left ventricular function and pulmonary artery pressure in patients with bronchiectasis. Chest 2008;133(2):468-473.

3. Barker AF. Bronchiectasis. N Engl J Med 2002;346(18):13831393.

4. Seitz AE, Olivier KN, Adjemian J, Holland SM, Prevots $\mathrm{R}$. Trends in bronchiectasis among medicare beneficiaries in the United States, 2000 to 2007. Chest 2012;142(2):432-439.

5. Dupont M, Gacouin A, Lena H, et al. Survival of patients whit bronchiectasis after the first ICU stay for respiratory failure. Chest 2004;125(5):1815-1820.

\section{$\triangle$ Correspondencia:}

Dr. Gustavo Lugo Goytia

Unidad de Cuidados Intensivos Respiratorios, Instituto Nacional de Enfermedades Respiratorias Ismael Cosío Villegas. Calzada de Tlalpan

Núm. 4502, Colonia Sección XVI, 14080, México, D.F. Correo electrónico: lugogoy@yahoo.com.mx

Los autores declaran no tener conflicto de intereses. 